\title{
Partial Presentation of an Extinction Cue Enhances Renewal in an ABA Paradigm
}

\author{
Pauline Dibbets*, Remco C. Havermans and Arnoud Arntz
}

Department Clinical Psychological Science, Maastricht University, P. O. Box 616, 6200MD, Maastricht, The Netherlands

\begin{abstract}
It was investigated in humans whether the incorporation of an extinction cue attenuates renewal of extinguished conditioned performance using a computerized task. All participants received an acquisition phase against a specific background, context A. In this context they learned that two conditioned stimuli, CS1 and CS2, predicted the occurrence of an aversive unconditioned stimulus (US) and that a third stimulus, CS3, predicted the absence of the US. Next, half of the participants received an extinction treatment in a different context (B), whereas the other half did not (context A). During extinction both CS1 and CS3 were repeatedly presented in absence of the US. For all participants, a so-called extinction cue was presented partially during this extinction phase. Finally, a renewal test was conducted in which CS1, CS2, and CS3 were presented against the acquisition background (A). Half of the participants received the extinction cue along the renewal test. Participants demonstrated renewal, but only when they had received the extinction treatment in a different context. Contrary to the hypothesis, the extinction cue at test did not attenuate but appeared to augment this renewal. Different explanations for this result are discussed.
\end{abstract}

Keywords: Fear conditioning, context, extinction, extinction cue, learning, renewal, retrieval.

\section{INTRODUCTION}

Pavlovian (or classical) fear conditioning in animals refers to the learning of an association between an initially neutral stimulus and a relevant, aversive event, the unconditioned stimulus (US). As a result of this learning, the initially neutral stimulus becomes a conditioned stimulus (CS) and will evoke a conditioned fear response (CR). It has been considered that anxiety disorders, like phobia, can be acquired through Pavlovian fear conditioning when an initially neutral stimulus (e.g. mouse) is paired with a fear evoking event (e.g. intense pain), resulting in a conditioned fear response to the CS $[1,2]$. This Pavlovian conditioning perspective on the aetiology of anxiety disorders resulted in a treatment procedure of great clinical relevance; the attenuation of a conditioned fear response by means of extinction. During extinction the CS is repeatedly presented in absence of the US. In a typical behaviour therapy session, patients are also exposed to the feared stimulus until the conditioned fear response is extinguished or strongly diminished [3-6].

Although extinction or exposure sessions do result in an attenuation of the conditioned fear response, it is well established that the original CS-US association is not erased [7, 8]. Retention of the CS-US association after extinction treatment can be uncovered in several ways including the mere passage of time (spontaneous recovery), fast relearning of the CS-US association (rapid reacquisition), unsignaled US presentations (reinstatement), or a change of context (renewal).

*Address correspondence to this author at the Department Clinical Psychological Science, Maastricht University, P. O. Box 616, 6200MD, Maastricht, The Netherlands; Tel: 0031 433881597; Fax: 0031 4338814155;

E-mail: Pauline.Dibbets@maastrichtuniversity.nl
Bouton states that extinction results in an additional association, the CS-noUS association, next to the already established CS-US association, leaving the CS with two meanings $[7,8]$. Which of the two available meanings is retrieved from memory depends on the context in which the CS is presented. Although both the CS-US and CS-noUS association are stored in memory, retrieval of the latter, second-learned association, is more vulnerable to changes in context than the former. This context dependency of extinction is most obvious in renewal. In the most common renewal paradigm, ABA renewal, the acquisition of the CSUS association takes place in one context (context A) and extinction is conducted in a separate context (context B). When the CS is then presented in the original conditioning context (context A) renewed responding is observed. Translated to a clinical setting a patient might acquire spider phobia at home (context A), receive exposure therapy in a clinic (context B), and suffer a relapse when confronted with a spider at home (context A)[9-12].

There are several ways to reduce a possible renewal effect after an extinction procedure or exposure therapy [7, 13, 14]. For instance, renewal is attenuated when extinction is conducted in multiple contexts [15], or when an extinction retrieval cue is added during extinction. Especially, the latter method seems to be a promising candidate for generalizing extinction beyond the extinction context. Brooks and Bouton [16] demonstrated in a series of animal conditioning experiments that presentation of a cue from extinction during renewal testing diminished the renewal effect. The authors state that this extinction cue helps to disambiguate the meaning of the CS during the renewal test by retrieving the memory of extinction. 
In our previous studies, we examined the influence of extinction cues on renewal using a human fear conditioning paradigm $[17,18]$. In these studies, acquisition took place in context $\mathrm{A}$, extinction in either context $\mathrm{A}$ or $\mathrm{B}$, and testing was conducted in context A. At test, half of the participants received an extinction cue that was also present during the extinction phase; the remaining participants did not receive this cue. The results demonstrated that a switch in context after extinction resulted in a renewed expectancy of the US on CS presentations (ABA). Most importantly, this renewal was attenuated by the presence of an extinction retrieval cue. Unexpectedly, removal of the cue in the group that did not receive a context switch (AAA) also resulted in renewed responding, indicating that the cue functioned as a conditioned inhibitor rather than a retrieval cue. In case of a conditioned inhibitor the existing CS-US association is suppressed and no, or only a weak, CS-noUS association is formed. Theoretically, withdrawal of the cue will then directly result in the activation of the CS-US association [19]. This observation implies that the fear is not extinguished, but only suppressed by the cue.

Therefore, the primary aim of the current study was to develop a method to encourage the retrieval properties of an extinction cue and, at the same time, to promote the formation of a CS-noUS association during extinction. In the animal experiments of Brooks and Bouton [16], the extinction cue was only partially presented during the extinction phase. This partial presentation still resulted in attenuation of renewal, whereas removal of the cue in the groups that did not receive a context switch did not result in renewed responding. Partial presentation of the cue during extinction has the advantage that a CS-noUS association can be formed on the no cue trials. To our knowledge, the usage of a partial presentation of an extinction retrieval cue to attenuate renewal has not been examined in humans.

For the present study, it was hypothesized that renewal after a switch in context should be reduced by the presentation of an extinction cue, even if this cue was only partially presented during the extinction phase. Additionally, it was hypothesized that removal of this cue after extinction should not result in renewed responding when no change in context was experienced.

\section{METHOD}

\subsection{Participants}

Eighty-four psychology and mental health students (46 females, 38 males) aged $18-29$ years $(M$ age $=21.18$ years, $S D=1.89)$ participated and received one hour of credit for their contribution. All participants signed a written informed consent before onset of the experiment, and were randomly assigned to one of four experimental groups, with the restriction of an equal male/female distribution in each group. The experiment was approved by the local ethical committee (ECP-66).

\subsection{Apparatus}

Three different photos of neutral faces, a white female, a white male, and a female with a dark skin colour (base 12 $\mathrm{cm}$, height $16 \mathrm{~cm}$ ) functioned as conditioned stimuli. A loud scream (2000 ms, $95 \mathrm{~dB}$, instantaneous rise time) was binaurally presented through headphones and served as unconditioned stimulus [20]. Two CSs were consistently paired with the US (CS1+ and CS2+); the remaining stimulus was never followed by the US (CS3-). Each photo functioned as CS1, CS2, and CS3 at least four times within each experimental group. For sake of clarity only one version will be described for the remainder of the article. The stimuli were presented against one of two different contexts (counterbalanced, only one version will be described below), an orange or a blue coloured computer screen. In all groups an ampersandsymbol " \&" functioned as the extinction retrieval cue. The US-expectancy was measured on each trial via a $100 \mathrm{~mm}$ online visual analogue scale (VAS.) The scale was presented at the bottom of the screen below the stimuli and the background. The indicator could be set anywhere between the far left (certainly no scream) and the far right end (certainly a scream) by clicking the left mouse button. The whole experiment was run on an IBM-compatible desktop computer and programmed with E-prime software (psychology Software Tools, http://www.pstnet.com).

\subsection{Procedure}

The experiment consisted of three phases: an Acquisition phase, an Extinction phase, and a Renewal test. Table 1 summarizes the experimental design. The four experimental groups were AAAnocue, AAAcue, ABAnocue, and ABAcue. The characters A and B refer to different contexts (orange and blue background colour) during the Acquisition, Extinction, and Renewal test, respectively. Cue indicates the presence or absence of the extinction cue at the Renewal test. For example, ABAnocue indicates that this group received the Acquistion in context A, Extinction in context B, and the Renewal test in context A. For this group no extinction cue was presented during the Renewal test.

\subsubsection{Pre-Conditioning}

After entering the room the participant was seated in a comfortable armchair in a quiet room. The experimenter was present in an adjacent room and communication was possible through an intercom system. Before onset of the experiment a questionnaire was presented concerning the stimuli used. For each of the three faces, the participant had to rate its (un)safety and (un)pleasantness on separate paper VASs. After these ratings the experimenter started the experiment and an instruction appeared on the computer screen. In this instruction the participant was encouraged to find the contingency between the photos presented and the (non)occurrence of a loud scream. Furthermore, information about the VAS usage was presented. Subsequently, the participant received three practice trials, without US presentations, in order to become customized to the rating procedure. Next, the US was presented twice and the participant rated the (un)pleasantness of the USs. The participant was told that the US should be loud and aversive, but not painful. If necessary, the volume of the US was adjusted on directions of the participant. The experimenter checked if all instructions were clear and then left the room. 
Table 1. Experimental Design

\begin{tabular}{|c|c|c|c|}
\hline Group & Acquisition & Extinction & Renewal Test \\
\hline \hline AAAnocue & $\mathrm{A}[\mathrm{CS} 1+]$ & $\mathrm{A}[\mathrm{CS} 1-] \mathrm{cue}$ & $\mathrm{A}[\mathrm{CS} 1]$ \\
& $\mathrm{A}[\mathrm{CS} 2+]$ & $\mathrm{A}[\mathrm{CS} 3-] \mathrm{cue}$ & $\mathrm{A}[\mathrm{CS} 3]$ \\
& $\mathrm{A}[\mathrm{CS} 3-]$ & & $\mathrm{A}[\mathrm{CS} 1] \mathrm{cue}$ \\
\hline AAAcue & $\mathrm{A}[\mathrm{CS} 1+]$ & $\mathrm{A}[\mathrm{CS} 2] \mathrm{cue}$ \\
& $\mathrm{A}[\mathrm{CS} 2+]$ & $\mathrm{A}[\mathrm{CS} 1-] \mathrm{CS} 3] \mathrm{cue}$ \\
\hline ABAnocue & $\mathrm{A}[\mathrm{CS} 3-]$ & $\mathrm{A}[\mathrm{CS} 3-] \mathrm{cue}$ & $\mathrm{A}[\mathrm{CS} 1]$ \\
& $\mathrm{A}[\mathrm{CS} 1+]$ & $\mathrm{A}[\mathrm{CS} 2]$ \\
& $\mathrm{A}[\mathrm{CS} 2+]$ & $\mathrm{B}[\mathrm{CS} 1-] \mathrm{cue}$ & $\mathrm{A}[\mathrm{CS} 3]$ \\
\hline ABAcue & $\mathrm{A}[\mathrm{CS} 3-]$ & $\mathrm{B}[\mathrm{CS} 3-] \mathrm{cue}$ & $\mathrm{A}[\mathrm{CS} 1] \mathrm{cue}$ \\
& $\mathrm{A}[\mathrm{CS} 1+]$ & $\mathrm{A}[\mathrm{CS} 2] \mathrm{cue}$ \\
& $\mathrm{A}[\mathrm{CS} 2+]$ & $\mathrm{B}[\mathrm{CS} 1-] \mathrm{cue}$ & $\mathrm{A}[\mathrm{CS} 3] \mathrm{cue}$ \\
\hline
\end{tabular}

Note: A and B represent the two different background colours, CS1, CS2, CS3 are the three conditioned stimuli, + followed by the US, - not followed by the US. Cue means the presence of the extinction cue. Testing was conducted without US.

\subsubsection{Acquisition Phase}

The acquisition phase was identical for all four groups. During the entire phase the background colour remained orange. At the bottom of the computer screen a grey band was presented, displaying the VAS. At the outer left anchor of the VAS the text "certainly not" was presented, indicating that no scream was expected, at the outer right the text "certainly" was displayed, indicating that the scream was certainly expected. The VAS was only displayed during CS presentation and only responses made within this time frame were recorded. The CS was presented in the centre of the coloured background and remained visible for 3 seconds. In case of CS1 and CS2, presentation of the US (2 seconds) followed immediately after offset of the CS. CS3 was never followed by the US. For all phases the inter-trial-interval varied between 6 and 8 seconds. Each CS was presented 6 times, resulting in a total of 18 trials. Stimuli were pseudorandomly presented with the restriction that a CS was never presented more than two times in succession and that UStrials were never presented more than four times in a row. The Extinction phase started automatically after the last acquisition trial.

\subsubsection{Extinction Phase}

For the $\mathrm{ABAnocue}$ and $\mathrm{ABAcue}$ group, the background colour switched to blue, for the AAAcue and AAAnocue group the background colour remained orange. For all groups, CS1 and CS3 were presented without US. Each stimulus was presented 12 times resulting in a total of 24 trials. Trials were pseudo-randomly mixed with the restriction that a stimulus was not presented more than three times in a row. The extinction cue was presented on $66.7 \%$ of the trials (i.e., 16 out of 24 trials). The position of the cue varied across trials, but the cue never covered the CS. Change in position occurred 4 seconds after offset of the CS and was introduced to discourage configural learning and to enhance the saliency of the extinction cue. After the last extinction trial, the Renewal test was automatically started.

\subsubsection{Renewal Test}

All groups received the Renewal test in the orange acquisition context. For half of the groups the extinction cue was present (AAAcue and ABAcue), for the other half no cue was present (AAAnocue and ABAnocue). Each stimulus was presented once. CS2 was presented in order to test transfer or generalization of the cue to a non-extinguished stimulus. Further details were identical to that of the extinction phase.

After finishing the experiment, the participant was asked to rate each CS again for (un)safety and (un)pleasantness. Furthermore, the US (un)pleasantness was rated and whether the amount of fear elicited by the US changed across trials (i.e. less fear or more fear).

\subsubsection{Inclusion Criteria}

Only the data of participants that mastered the Acquisition and Extinction phase were included in the data analyses. Performance was labelled as successful if the following criteria were met: 1) a rating of $80 \%$ or more on the last CS1 and CS2 acquisition trial, indicating a clear expectancy of the US; 2 ) a rating of $20 \%$ or less on the last CS3 acquisition trial, indicating that no US was expected; 3 ) a rating of $20 \%$ or less on the last CS1 and CS3 ratings, indicating successful extinction and the maintenance of no US expectancy, respectively. These criteria were set as testing for potential renewal is redundant in the absence of adequate acquisition and extinction.

\subsection{Response Definition}

The amount of renewal was calculated by subtracting the ratings of CS1 on the last extinction trial, trial 12, from the ratings of the Renewal test. A similar difference score was calculated for CS3. For CS2 the difference between the last acquisition trial, trial 6 , and the test rating was calculated. 


\subsection{Statistical Analyses}

Both the US expectancy data and pleasantness and safety ratings were parametrically analyzed using ANOVAs and repeated measures ANOVAs. Context (same versus switch) and cue (present or absent at test) served as between-subjects factors. Effects sizes were expressed as partial eta squared, $\mathrm{p}^{2}$. In case of violations of sphericity, Greenhouse Geisser corrections were made; Bonferroni corrections were made in case of multiple or pair-wise comparisons. The significance level was set at $p<.05$, throughout.

\section{RESULTS}

The data from 23 students were discarded because they did not meet one or more of the aforementioned criteria. Seventeen of these participants were excluded because of unsuccessful acquisition, six because of unsuccessful extinction. The remaining 61 students were 32 females and 29 males $(M$ age $=21.03$ years; $S D=1.83)$. The number of participants in each group was: AAAnocue $n=16$, AAAcue $n=14$, ABAnocue $n=16$, ABAcue $n=15$. No difference between the four experimental groups concerning age, $F<1$, or gender, $\chi^{2}<1$, was detected.

\subsection{Acquisition}

The mean US expectancy ratings of CS1, CS2, and CS3 are depicted on the left side of Fig. (1). A repeated measures ANOVA with stimulus type (CS1, CS2, and CS3) and trial (1 through 6) as within-subjects factors and cue and context as between-subjects factors was used to analyze the US expectancy ratings of the acquisition phase. This analysis re- vealed a main effect of stimulus, $F(2,114)=624.93, p<$ $.001, \mathrm{p \eta}^{2}=.92$, a main effect of trial, $F(5,285)=49.88, p<$ $.001, \mathrm{p}^{2}=.47$, but no main effect of context or cue, $F \mathrm{~s}(1$, $57)<1.55, \mathrm{ps}>.22, \mathrm{p \eta}^{2}<.027$. Additionally, a stimulus $\mathrm{x}$ trial interaction was observed, $F(10,570)=81.66, p<.001$, $\mathrm{p}^{2}=.59$, and a significant stimulus $\mathrm{x}$ context $\mathrm{x}$ cue interaction, $F(2,114)=3.78, p=.039, \mathrm{p}^{2}=.062$. No other significant interaction effects were observed, $F \mathrm{~s}<3.08, p \mathrm{~s}>.077$, $\mathrm{p}^{2}=<.052$.

Firstly, the stimulus $\mathrm{x}$ trial interaction was analyzed further by comparing CS1, CS2, and CS3 on each of the six acquisition trials using repeated measures ANOVAs. These analyses revealed a main effect of stimulus on each trial, $F \mathrm{~s}(2,120)>3.48, p \mathrm{~s}<.037$, with an increase in effect size as the acquisition phase progressed $\left(.054<\mathrm{p \eta}^{2}<.99\right)$. On trial $1 \mathrm{CS} 1$ received lower ratings than $\mathrm{CS} 2, p=.019$; no other differences were observed, $p \mathrm{~s}>.25$. On the remaining trials $\mathrm{CS} 1$ and CS2 received higher ratings than did CS3, ps < .001 , with no differences between CS1 and CS2, $p \mathrm{~s}>.34$.

Secondly, the stimulus $\mathrm{x}$ context $\mathrm{x}$ cue interaction was further examined by averaging each CS across the six acquisition trials and running Univariate Analyses of Variance on these averages with group as factor (i.e. splitting up cue/no cue and context switch/no switch). Only for CS1 a main effect of group was observed, $F(3,57)=3.76, p=.016, \mathrm{p \eta}^{2}$ $=.17$, but not for $\mathrm{CS} 2$ and $\mathrm{CS} 3, F \mathrm{~s}(3,57)<1.87, p \mathrm{~s}>.12$, $\mathrm{pq}^{2}<.095$. Pairwise comparisons indicated that, overall, the AAAnocue group displayed higher CS1 ratings than did the ABAnocue group, $p=.10$, no other group differences were observed, $p \mathrm{~s}>.29$. This was not considered as being problematic as no group differences were detected on the last

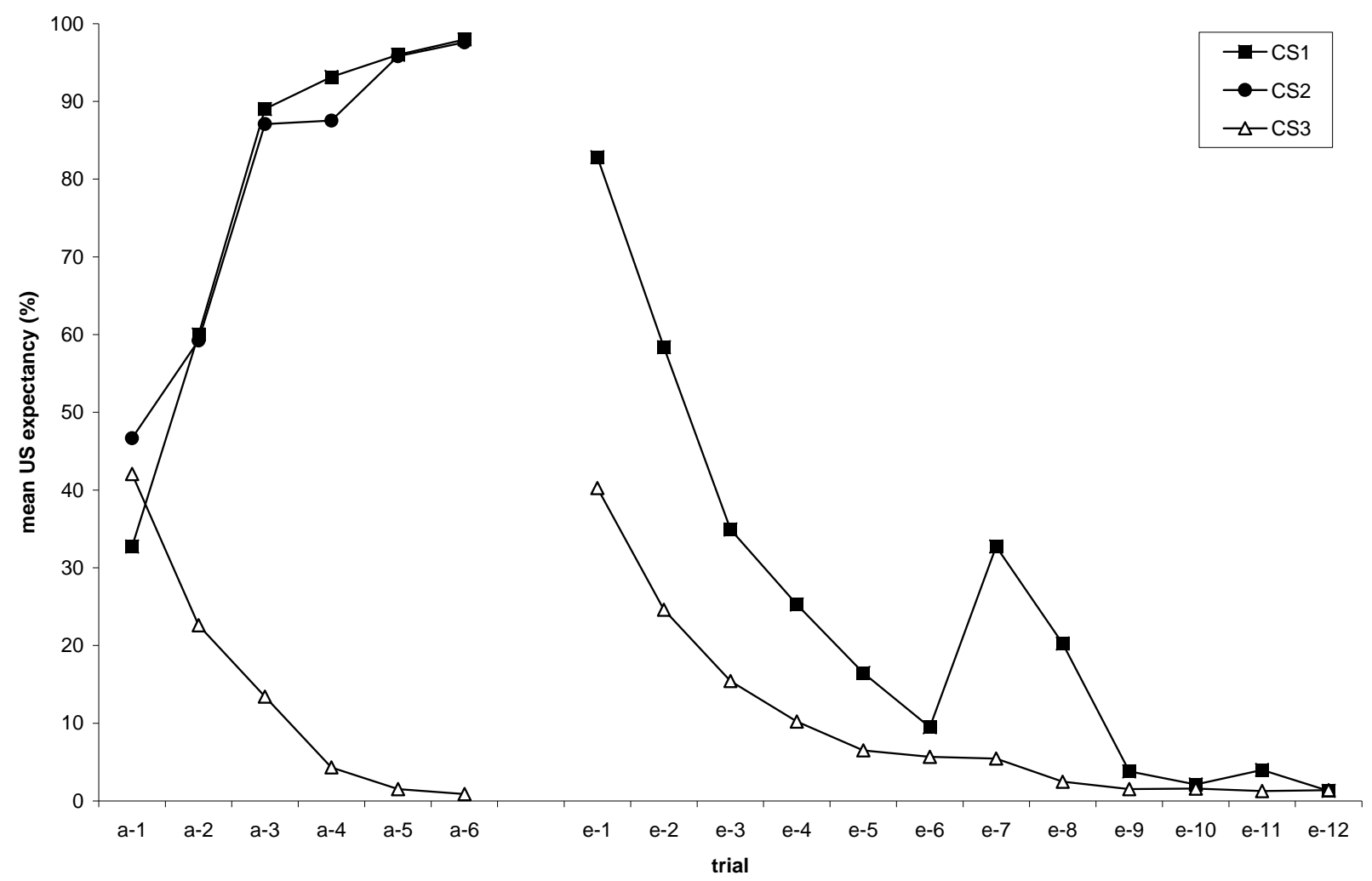

Fig. (1). Acquisition of the CS1, CS2, and CS3 expectancy ratings, and extinction of the CS1 and CS3 expectancy ratings. 
CS1 acquisition trial, $F(3,60)=1.00, p=.40, \mathrm{p}^{2}=.050$. As can be derived from Fig. (1), CS1 and CS2 ratings gradually increased as the acquisition phase progressed, whereas CS3 ratings steadily declined.

\subsection{Extinction}

The extinction ratings are displayed on the right side of Fig. (1). As can be inferred from this figure, the ratings of CS1 on the first extinction trial appear to be smaller than that of the final acquisition trial, whereas the ratings of CS3 appear to have increased. This suggests some generalization decrement due to a switch in context. To test this assumption, two separate repeated measures ANOVAs were conducted on CS1 and CS3. In each analysis the last acquisition trial and first extinction trial served as within-subjects factor and context switch (AAA-groups versus ABA-groups) functioned as between-subjects factor. Both analyses revealed a main effect of trial moment, $F \mathrm{~s}(1,59)>13.46$, ps $<.002$, $\mathrm{p}^{2}$ $>.18$, a main effect of context switch, $F \mathrm{~s}(1,59)>13.68$, $p \mathrm{~s}$ $<.001, \mathrm{p \eta}^{2}>.18$, and a trial moment $\mathrm{x}$ context switch interaction, $F_{\mathrm{s}}(1,59)>13.73, p \mathrm{~s}<.001, \mathrm{p \eta}^{2}>.18$. Paired t-tests indicated that CS1 ratings increased in case a switch took place, $t(30)=3.75, p=.001$, but not in case the context remained the same, $t<1$. For both the switch and the non switch condition CS3 ratings increased, $t s>2.64, p s<.014$, but with a sharper increase in case of a context switch, $t(59)$ $=5.12, p<.001$. The change in ratings of CS1 and CS3 in the switch group can easily be explained by the clear transition from the acquisition to the extinction phase. For these groups the background colour changed. Furthermore, for all groups CS1 was no longer followed by the US, indicating that the contingencies learned were no longer valid. It appears that this change was also noticed in the AAA-groups, resulting in a small increase of the CS3 ratings, indicating that the participants were no longer entirely confident that no US would follow.

A GLM repeated measures was conducted to further analyze the extinction data. In this analysis stimulus (CS1 and CS3) and trial (1 through 12) served as within-subjects factors and cue and context switch as between-subjects factors. This analysis revealed a main effect of stimulus, $F(1$, $57)=59.69, p<.001, \mathrm{p}^{2}=.51$, a main effect of trial, $F(11$, $627)=84.65, p<.001, \mathrm{p}^{2}=.60$, but no main effect of cue or context switch, $F \mathrm{~s}(1,57)<1.25, \mathrm{ps}>.26, \mathrm{p}^{2}<.023$. Furthermore, a stimulus $\mathrm{x}$ trial interaction was observed, $F(11,627)=14.93, p<.001, \mathrm{p}^{2}=.21$, a stimulus $\mathrm{x}$ context switch interaction, $F(1,57)=37.83, p<.001, \mathrm{p \eta}^{2}=.40$, a trial $\mathrm{x}$ context switch interaction, $F(11,627)=3.68, p=$ .003 , a trial $\mathrm{x}$ context switch $\mathrm{x}$ cue interaction, $F(11,62)=$ $3.68, p=.003, \mathrm{p}^{2}=.061$, and a stimulus $\mathrm{x}$ trial $\mathrm{x}$ context switch interaction, $F(11,627)=13.06, p<.001, \mathrm{p \eta}^{2}=.19$. The trial $\mathrm{x}$ cue interaction just failed to reach significance, $F(11,627)=2.07, p=.067, \mathrm{p}^{2}=.035$.

To further assess the interactions observed, separate GLM repeated measures were run on CS1 and CS3, with cue and context switch as between-subjects factors. Both analyses indicated that CS ratings decreased as extinction progressed, $F \mathrm{~s}(11,627)>26.71, p \mathrm{~s}<.001, \mathrm{p}^{2}>.31$, but with a sharper decrease for CS1 than for CS3 (i.e. extinction trial 1 minus 12 ), paired t-test, $t(60)=5.72, p<.001$. Both analyses revealed a main effect of context switch, $F_{\mathrm{s}}(1,57)>14.45$, $p \mathrm{~s}<.001, \mathrm{p \eta}^{2}>.20$, and a trial $\mathrm{x}$ context switch interaction, $F \mathrm{~s}(11,627)>6.23, p \mathrm{~s}<.001, \mathrm{p \eta}^{2}>.098$. Additionally, for CS3 a trial $\mathrm{x}$ context switch $\mathrm{x}$ cue interaction was observed, $F(11,627)=3.36, p=.012, \mathrm{p}^{2}=.056$. No other (interaction) effects were observed, $F \mathrm{~s}<1.47, p \mathrm{~s}>.21, \mathrm{p}^{2}<.026$.

The CS1 trial $\mathrm{x}$ context switch interaction was assessed further by running additional Univariate Analyses of Variance on each trial with context switch as factor. These analyses revealed that a switch in context resulted in lower ratings on trial 1 through 3 , and trial 7 and $8, F \mathrm{~s}(1,59)>10.98$, ps $<$ $.025, \mathrm{p}^{2}>.15$. No such context effect was observed on the remaining trials, $F \mathrm{~s}(1,59)<1.25, \mathrm{ps}>.27, \mathrm{p \eta}^{2}<.022$.

The CS3 trial $\mathrm{x}$ context switch $\mathrm{x}$ cue interaction was analyzed further by running separate Univariate Analyses of Variance on each trial with group (i.e. dividing cue/no cue and context switch/no switch) as factor. These analyses revealed that the group differences were only observed on the first two trials, $F \mathrm{~s}(3,57)>6.51, p \mathrm{~s}<.013, \mathrm{p \eta}^{2}>.25$, but not on the remaining trials, $F_{\mathrm{s}}(3,57)<2.43, \mathrm{ps}>.074, \mathrm{p \eta}^{2}<.12$. Post Hoc tests indicated that on trial 1 the AAAcue group significantly differed from the ABAcue and ABAnocue groups, $p$ s $<.008$, but not from the AAAnocue group, $p=$ 1.00. The AAAnocue group also differed from the ABAcue group, $p=.001$, and a marginally significant effect was observed between the AAAnocue and ABAnocue group, $p=$ .068. For trial 2, a difference between the ABAcue and AAA-groups was observed, $p \mathrm{~s}<.005$. Overall, a switch in context resulted in higher US expectancies on presentation of CS3 than no switch in context, especially visible at the onset of the extinction phase.

\subsection{Renewal Test}

The mean ratings of CS1, CS2, and CS3 on the Renewal test are displayed in Fig. (2). The amount of renewal for CS1 was calculated by subtracting the ratings on the last extinction trial from the Renewal test rating. A GLM repeated measures with stimulus as within-subjects factor and cue and context switch as between-subject factors was carried out. This analysis revealed a main effect of stimulus, $F(2,114)=$ 105.73, $p<.001, \mathrm{p \eta}^{2}=.65$, and a main effect of context switch, $F(1,57)=24.13, p<.001, \mathrm{p \eta}^{2}=.30$. Additionally, a stimulus $x$ context switch interaction, $F(2,114)=21.58, p<$ $.001, \mathrm{p}^{2}=.28$, and a context switch $\mathrm{x}$ cue interaction, $F(1$, $57)=4.30, p=.043, \mathrm{p \eta}^{2}=.070$, were observed. Pairwise comparisons indicated that $\mathrm{CS} 2$ received the highest ratings followed by CS1 and CS3, $p$ s $<.001(\mathrm{CS} 2>\mathrm{CS} 1>\mathrm{CS} 3)$.

To examine the observed interaction, separate Univariate Analyses of Variance with this difference score as dependent variable and cue and context switch as factors were carried out, followed by pairwise comparisons between all groups. For CS1 this analysis revealed a main effect of context switch, $F(1,57)=74.78, p<.001, \mathrm{p \eta}^{2}=.57$, a marginally significant effect of cue, $F(1,57)=3.46, p=.068, \mathrm{p}^{2}=$. 057 , and a significant cue $\mathrm{x}$ context switch interaction, $F(1$, $57)=4.95, p=.030, \mathrm{p \eta}^{2}=.08$. The presence of a context switch resulted in more renewal. Although not significant, the presence of the cue tended to result in more renewal. This was contrary to our expectations and previouslyobtained results using a similar experimental design. The 
interaction was caused by a diversion in the effect of the cue on CS1 responding between the AAA- and ABA-groups. Multiple comparisons indicated that AAAcue and AAAnocue group did not differ from each other, $p=1.00$, that the two ABA-groups showed more renewal than both AAA-groups, $p s<.001$, and that the ABAcue group displayed more renewal than the ABAnocue group, $p=.03$. The amount of renewal can be summarized as follows: ABAcue $>$ ABAnocue $>$ AAAcue $=$ AAAnocue. Pairwise comparisons between the last extinction trial and first test trial revealed only an increase in CS1 ratings in both ABA groups, $p$ s $<.001$, no change was detected in the AAAgroups, $p s>.11$.

Secondly, the influence of a cue and a switch in context on CS2 responding was analyzed. The difference score was calculated by subtracting the last CS2 acquisition ratings, trial 6, from the Renewal test ratings. This analysis revealed no effect of cue, context switch or cue $x$ context switch interaction, $F \mathrm{~s}<2.90, \mathrm{ps}>.093, \mathrm{p}^{2}<.049$. These results indicate that the cue did not transfer its value to another, non-extinguished, stimulus. Pairwise comparisons between the last acquisition trial and first CS2 test trial revealed a decrease in ratings for all groups, $p \mathrm{~s}<.05$.

Finally, a similar analysis was run on the CS3 difference score. This analysis revealed only a main effect of context switch, $F(1,57)=18.32, p<.001, \mathrm{p \eta}^{2}=.24$, but no main effect of cue or cue $\mathrm{x}$ context switch interaction, $F \mathrm{~s}<1$. Pairwise comparisons of the groups revealed higher CS3 ratings, relative to the last extinction trial, for each of $\mathrm{ABA}$ groups compared to each of the AAA-groups, $p \mathrm{~s}<.05$. No differences between the two AAA- and two ABA-groups were observed, $p \mathrm{~s}=1.00$. Additional comparisons revealed that for both $\mathrm{ABA}$ groups CS3 ratings significantly increased from the last extinction trial to the first test trial, $p \mathrm{~s}<.05$, but no such increase was observed in the AAA groups, $p s>.13$.

\subsection{Ratings of the Stimuli}

\subsubsection{Pleasantness}

The mean ratings and accompanying standard errors of each stimulus are presented in Table 2. A GLM repeated measures was conducted to assess the ratings of the three CSs before onset of the experiment. In this analysis CS1, CS2, and CS3 functioned as within-subjects factor and cue and context switch as between-subjects factors. This analysis revealed no effect of stimulus, cue, context or interactions, $F_{\mathrm{s}}<1.24, p \mathrm{~s}>.29, \mathrm{p \eta}^{2}<.022$. These results indicate that at the onset of the experiment no differences in experienced pleasantness were observed.

A similar GLM was run on the pleasantness ratings after the experiment. This analysis indicated a main effect of stimulus, $F(2,114)=8.82, p<.001, \mathrm{p \eta}^{2}=.13$, but no main or interaction effects of context switch or cue, $F \mathrm{~s}<1.42$, $p \mathrm{~s}$ $>.23, \mathrm{p}^{2}<.025$, Pairwise comparisons revealed that both $\mathrm{CS} 1$ and CS2 were rated as less pleasant than CS3, ps < .007 . No difference between CS1 and CS2 was obtained, $p=$ 1.00 .

\subsubsection{Safety}

The safety data were analyzed in the same way as the pleasantness ratings. Like the pleasantness ratings no differences or interaction effects were detected before the onset of the experiment, $F \mathrm{~s}<1.24, \mathrm{ps}>.29, \mathrm{p}^{2}<.22$. These results indicate similar safety ratings across stimuli and groups.

Renewal Test

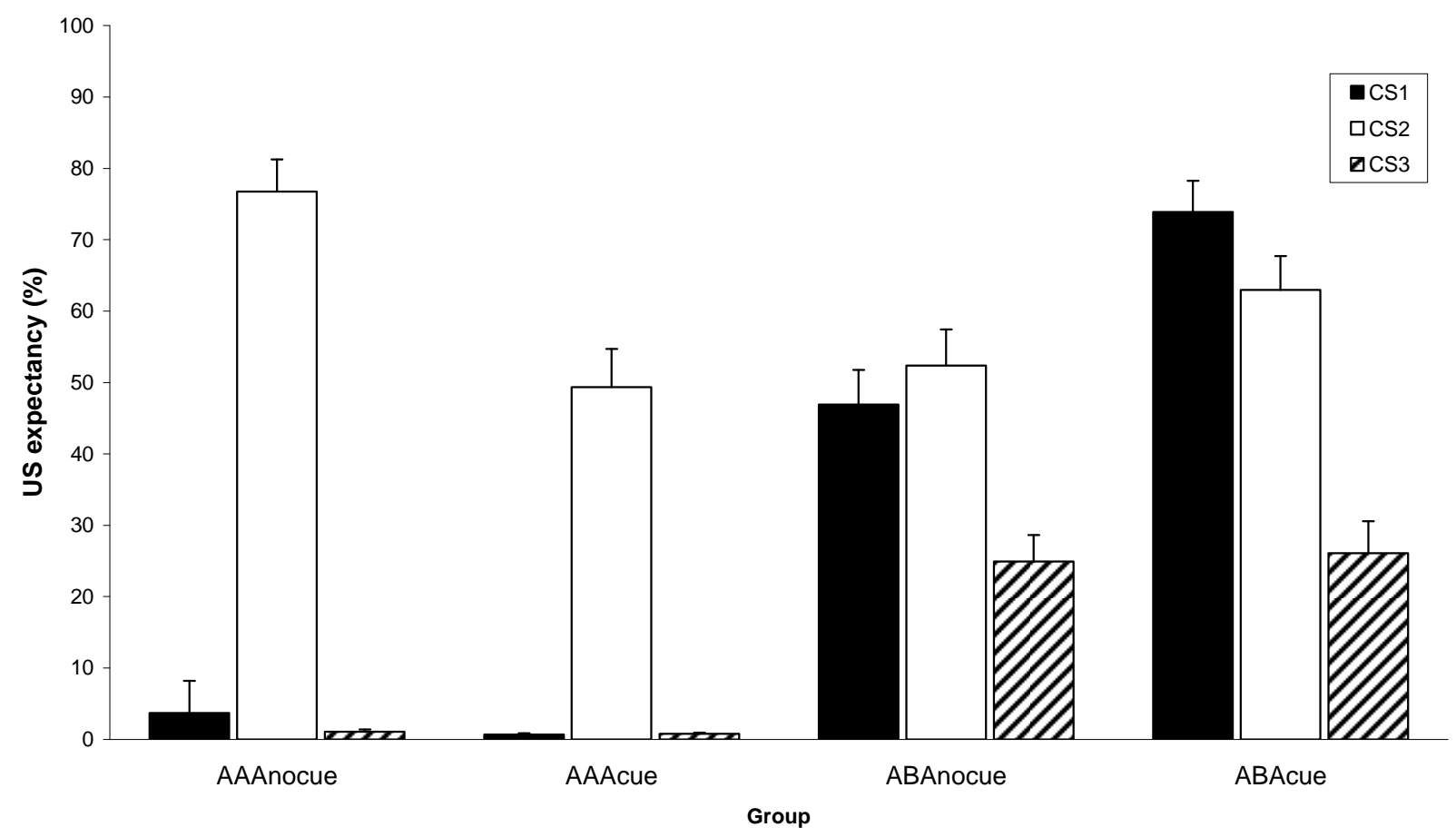

Fig. (2). The mean ratings of CS1, CS2, and CS3 on the Renewal test. 
Table 2. Stimulus Ratings

\begin{tabular}{|c|c|c|c|c|c|c|c|c|}
\hline \multicolumn{9}{|c|}{ Ratings Before Experiment } \\
\hline \multirow{2}{*}{ Group } & \multicolumn{2}{|c|}{ CS1 } & \multicolumn{2}{|c|}{ CS2 } & \multicolumn{2}{|c|}{ CS3 } & \multirow{2}{*}{$\begin{array}{c}\text { US } \\
\text { Pleasant }\end{array}$} & \\
\hline & Pleasant & Safe & Pleasant & Safe & Pleasant & Safe & & \\
\hline \multirow[t]{2}{*}{ AAAnocue } & 44.44 & 56.19 & 45.38 & 58.00 & 52.13 & 60.44 & 12.88 & \\
\hline & 4.83 & 6.61 & 6.34 & 6.41 & 4.51 & 6.66 & 2.37 & \\
\hline \multirow[t]{2}{*}{ AAAcue } & 46.50 & 55.07 & 57.43 & 67.57 & 50.29 & 58.93 & 14.71 & \\
\hline & 2.83 & 5.49 & 5.18 & 5.76 & 4.74 & 5.67 & 2.43 & \\
\hline \multirow[t]{2}{*}{ ABAnocue } & 50.81 & 60.25 & 52.75 & 65.69 & 47.00 & 61.75 & 15.31 & \\
\hline & 6.46 & 5.59 & 4.33 & 5.04 & 5.09 & 3.50 & 3.19 & \\
\hline \multirow[t]{2}{*}{ ABAcue } & 50.07 & 60.67 & 55.33 & 60.33 & 53.13 & 64.13 & 13.20 & \\
\hline & 3.30 & 5.75 & 5.34 & 5.86 & 3.85 & 4.91 & 2.03 & \\
\hline & \multicolumn{7}{|c|}{ Ratings After Experiment } & Change \\
\hline \multirow[t]{2}{*}{ AAAnocue } & 38.94 & 51.31 & 35.25 & 46.56 & 56.25 & 71.88 & 16.94 & 27.19 \\
\hline & 6.32 & 6.38 & 6.78 & 7.04 & 6.64 & 5.55 & 3.57 & 6.75 \\
\hline \multirow[t]{2}{*}{ AAAcue } & 42.29 & 48.93 & 52.00 & 56.57 & 53.64 & 64.07 & 24.36 & 28.57 \\
\hline & 5.76 & 7.34 & 5.48 & 7.11 & 4.16 & 4.92 & 4.00 & 7.64 \\
\hline \multirow[t]{2}{*}{ ABAnocue } & 44.69 & 51.50 & 39.44 & 46.19 & 47.19 & 55.31 & 20.56 & 19.87 \\
\hline & 7.24 & 6.95 & 5.92 & 6.38 & 5.76 & 6.39 & 4.15 & 5.18 \\
\hline \multirow[t]{2}{*}{ ABAcue } & 39.67 & 48.60 & 43.13 & 52.20 & 61.60 & 72.87 & 20.67 & 20.67 \\
\hline & 3.31 & 5.49 & 6.46 & 7.91 & 4.71 & 3.58 & 5.82 & 6.16 \\
\hline
\end{tabular}

Note: Mean ratings and standard errors of pleasantness and safeness of CS1, CS2, and CS3 before and after the experiment. The US is rated for (un)pleasantness and rated for (un)pleasantness and change in fear (change) after the experiment. In this table highly pleasant/safe/ is represented by scores close to 100 . In case of a change in fear a score close to 100 represents sharp increase in fear.

The GLM conducted on the safety ratings after finishing the experiment revealed a main effect of stimulus, $F(2,114)$ $=13.19, p<.001, \mathrm{p \eta}^{2}=.19$. No other main or interaction effects of cue or context switch were observed, $F \mathrm{~s}<1.19$, ps $>.30, \mathrm{p \eta}^{2}<.021$. Pairwise comparisons revealed that both $\mathrm{CS} 1$ and CS2 were rated as less safe than CS3, $p \mathrm{~s}<.001$. No difference between CS1 and CS2 was observed, $p=1.00$.

\subsubsection{US Ratings}

A Univariate Analyses of Variance on the US pleasantness rating revealed no effect of cue, context switch, or interaction, $F \mathrm{~s}<1$, before onset of the experiment. The US was perceived as highly unpleasant, one sample t-test, comparison to neutral value $50, t=28.62, p<.001$.

Similarly, after completing the experiment, no effect of context switch, cue or interaction was observed, $F \mathrm{~s}<1$. However, the unpleasantness of the US declined over the two measurement moments, $F(1,57)=13.31, p=.001, \mathrm{p}^{2}=$ .19 , and the amount of fear elicited by the US diminished, one sample t-test, comparison to no change value $50, t \mathrm{~s}>$ $2.80, p \mathrm{~s}<.05$. These results indicated that with repeated exposure, participants habituated to the US.

\section{DISCUSSION}

In the present experiment it was investigated whether a partially presented extinction retrieval cue can attenuate renewal after a switch in context. At the same time it was tested whether this partial presentation enabled the formation of a CS-noUS association during the extinction phase. The results indicated that most participants readily learned to differentiate between the CSs that predicted the occurrence of the US, CS1 and CS2, and the CS that predicted the nonoccurrence of the US, CS3. The resulting difference in USexpectancy between CS1 and CS3 was successfully extinguished in $90 \%$ of the participants in the subsequent extinction phase. At test, this differential US-expectancy was renewed when the test context differed from the extinction context (ABA groups). Contrary to our expectations, this renewal effect was not attenuated when the extinction cue was present. Even more, the presence of a cue after a switch in context resulted in more renewal than its absence. Furthermore, the cue did not attenuate the US expectancy of the non-extinguished CS2.

Next to the ability of an extinction cue to reduce renewed responding at test, the formation of a CS-noUS association during extinction was examined. To this end it was tested whether removal of the cue after extinction would result in a higher US expectancy on CS1 presentation. The results showed that the low US expectancy on CS1 presentation after cue removal was indeed preserved. That is, at test no difference between the AAAcue and AAAno cue group was observed, and compared to the extinction phase the CS1 
ratings at test remained equally low. This persistence in low ratings indicate that the cue did not merely function as a conditioned inhibitor that suppressed the CS - noUS association.

The valence ratings of the stimuli showed that after the experiment participants rated the CS3 as the most pleasant and safe stimulus. This comes as no surprise since CS3 was never followed by the US during the entire experiment. The US ratings pointed out that the aversion decreased during the experiment, indicating habituation to the loud scream.

The results of the present study are only partly in line with our expectations. Removal of a formally partially presented cue at test indeed did not produce renewed CS1 responding in the AAAnocue group. This result corresponds with previous animal findings [16]. However, contrary to the results of Brooks and Bouton the cue enhanced rather than diminished renewal in the ABA groups. Given the fact that such an augmentation was not hypothesized, further human research should first replicate the abovementioned animal findings before definitive conclusions can be drawn. A possible explanation for the discrepancy observed between our data and those of Brooks and Bouton is that we used a fear rather than an appetitive conditioning paradigm. In our paradigm we used stimuli that belonged together, a face with a loud aversive scream. Such combination is known to result in strong fear conditioning that is less vulnerable to extinction [20]. It is, therefore, conceivable that fear conditioning is more sensitive to renewal than appetitive conditioning. If that is the case, presentation of the extinction cue at test might not be strong enough to diminish this renewal. Although this line of reasoning can explain the absence of a diminishment in renewal by the retrieval cue, it cannot clarify the augmentation of renewal by its presence.

A second explanation is that the cue was not the best stimulus to signal the non-occurrence of the US. In the ABA groups the background colour was always present and, therefore, reliably predicted the absence of the US. The cue was only partially presented $(66.67 \%)$ and the absence of the US occurred both in its presence as well as in its absence. As a result the extinction cue was experienced as non-informative and attention to it waned [21]. At test, the cue was presented against a different background and evoked renewed attention. Because of its previous history, participants in the ABAcue group learned little or nothing about the cue-US relation, resulting in no attenuation of renewal. Although this explanation does not directly account for the increased CS1 ratings in the ABAnocue group, one can argue that presentation of the cue at test caused confusion resulting in a general increase in ratings. If this is the case, differential CS2 and CS3 ratings at test are also expected, but no differences between the ratings of the $\mathrm{ABAcue}$ and $\mathrm{ABAnocue}$ group were observed, $F \mathrm{~s}<1$

A final, and most fitting, explanation is that the cue indeed was not informative regarding the CS1-(no)US association and that its function remained unclear for the participants. This ambiguity only acted on other equivocal stimuli. In the present study only CS1 was endowed with an ambivalent meaning, predicting both the occurrence and nonoccurrence of the US. The remaining two CSs, CS2 and CS3, were consequently followed by the presence and absence of the US, respectively. One can argue that especially the combination of the two uncertain stimuli, that is CS1 and the extinction cue, elicited a higher US expectancy after a switch in context.

Besides these possible explanations for the diverging results between the present study and that of Brooks and Bouton [16], it is also important to note that the presentation scheme of the extinction cue differed. In the study of Brooks and Bouton [16] the cue preceded the non-reinforced CSs. This procedure is known to produce negative occasion setting in which the cue signals that in its presence the CS-US association is no longer valid [22]. As a result reduced responses on the extinguished CS are expected in its presence after a switch in context. In our study, the cue was presented throughout the entire trial and both preceded and accompanied the CSs. Holland [23] suggests that perceptual discontinuity between the stimuli, for example by establishing a time interval, favours occasion setting, whereas simultaneous presentation is more likely to result in conditioned inhibition. In the present study both situations are effective, the cue both preceded and accompanied the CS. Furthermore, the cue was not continuously present and changed position between trials, reducing the chance that it was merely an element of the context. This design might have provided the cue with both occasion-setting and conditioned inhibitor properties. Even if this is the case, both types of function should have resulted in decreased CS1 ratings at test.

In sum, the present results suggest that when a discrete cue is partially added to the extinction phase, the removal of such a cue does not automatically lead to renewed responding. These results concord with animal research in which an extinction cue did not merely functioned as a conditioned inhibitor and the formation of a CS-noUS association remained possible [24, 25]. Furthermore, adding the cue after a switch in context resulted in more renewal, probably due to the ambiguity of the CS1 and extinction cue combination. This latter effect was contrary to our expectations and previous results on human experiments using extinction cues $[17$, $26,27]$.

The current study has several limitations. First of all, a total of 23 students did not demonstrate clear acquisition $(n=$ 17 ) or extinction $(n=6)$. During acquisition, seven persons were excluded as they seemed to have made a 'mistake' or lost their attention. That is, when the 'incorrect' last rating was omitted and replaced the second last rating (acquisition trial 5) they did fulfil the inclusion criteria. Additionally, seven persons showed a moderate response style [28], with CS1 and CS2 rated well above CS3, but with expectancies below $80 \%$ or above $20 \%$. Six participants were excluded as they did not (fully) adjust their US expectancy ratings during the extinction phase, resulting in CS1 scores over $20 \%$. The remaining three participants did not seem to master the task at all.

Second, the participants habituated to the US. Such habituation of course affects conditioned fear responding and hinders a direct comparison with clinical research. Therefore, in future research, one should attempt to make use of a more aversive US, such as an electric shock [e.g., 26, but see 29] or synthesized aversive sounds such as fingernails scraping across a chalkboard [30] in order to reduce habituation. Sec- 
ond, although partial presentation of an extinction cue did allow the formation of a CS-noUS association, its applicability in a clinical setting is limited. The main aim of an extinction cue is to reduce rather than enhance renewal after exposure. Therefore, future research is necessary to optimize procedures that encourage the formation of a CS-noUS association during extinction, the attenuation of renewal after a switch in context, and the transfer of such a cue to other non-extinguished stimuli.

\section{ACKNOWLEDGEMENTS}

We would like to thank Shelley van Gerven, Len Hamers, and Renske Steenbakkers for their contribution to this research paper.

\section{CONFLICT OF INTEREST}

The author(s) confirm that this article content has no conflicts of interest.

\section{REFERENCES}

[1] Mowrer $\mathrm{OH}$. A stimulus-response analysis of anxiety and its role as a reinforcing agent. Psychol Rev 1939; 46(6): 553-65.

[2] Wolpe J. Psychotherapy by reciprocal inhibition. Stanford: Stanford University Press 1958.

[3] Marks IM, Hodgson R, Rachman S. Treatment of chronic obsessive-compulsive neurosis by in-vivo exposure: a two-year follow-up and issues in treatment. Br J Psychiatry 1975; 27: 34964.

[4] Öst LG. One-session treatment for specific phobias. Behav Res Ther 1989; 27(1): 1-7.

[5] Öst LG. One-session group treatment of spider phobia. Behav Res Ther 1996; 34(9): 707-15.

[6] Öst LG. Rapid treatment of specific phobias. In: Davey GCL, Ed. Phobias: a handbook of theory, research and treatment. Chichester, England: John Wiley \& Sons 1997; pp. 227-46.

[7] Bouton ME. Context, ambiguity, and unlearning: sources of relapse after behavioral extinction. Biol Psychiatry 2002; 52(10): 976-86.

[8] Bouton ME. Context and behavioral processes in extinction. Learn Mem 2004; 11(5): 485-94.

[9] Rodriguez BI, Craske MG, Mineka S, Hladek D. Contextspecificity of relapse: Effects of therapist and environmental context on return of fear. Behav Res Ther 1999; 37(9): 845-62.

[10] Mineka S, Mystkowski JL, Hladek D, Rodriguez BI. The effects of changing contexts on return of fear following exposure therapy for spider fear. J Consult Clin Psychol 1999; 67(4): 599-604.

[11] Mystkowski JL, Mineka S, Vernon LL, Zinbarg RE. Changes in caffeine states enhance return of fear in spider phobia. J Consult Clin Psychol 2003; 71(2): 243-50.
[12] Mystkowski JL, Craske MG, Echiverri AM. Treatment context and return of fear in spider phobia. Behav Ther 2002; 33(3): 399-416.

[13] Conklin CA, Tiffany ST. Applying extinction research and theory to cue-exposure addiction treatments. Addiction 2002; 97(2): 15567.

[14] Havermans RC, Jansen ATM. Increasing the efficacy of cue exposure treatment in preventing relapse of addictive behavior. Addict Behav 2003; 28(5): 989-94.

[15] Gunther LM, Denniston JC, Miller RR. Conducting exposure treatment in multiple contexts can prevent relapse. Behav Res Ther 1998; 36(1): 75-91.

[16] Brooks DC, Bouton ME. A retrieval cue for extinction attenuates response recovery (renewal) caused by a return to the conditioning context. J Exp Psychol Anim Behav Process 1994; 20(4): 366-79.

[17] Dibbets P, Havermans R, Arntz A. All we need is a cue to remember: the effect of an extinction cue on renewal. Behav Res Ther 2008; 46(9): 1070-7.

[18] Dibbets P, Maes JHR. The effect of an extinction cue on ABArenewal: does valence matter? Learn Motiv 2011; 42: 133-44.

[19] Lovibond PF, Davis NR, O'Flaherty AS. Protection from extinction in human fear conditioning. Behav Res Ther 2000; 38(10): 967-83.

[20] Hamm AO, Vaitl D, Lang PJ. Fear conditioning, meaning, and belongingness: a selective association analysis. J Abnorm Psychol 1989; 98(4): 395-406.

[21] Kamin LJ. Predictability, surprise, attention and conditioning. In: Campbell BA, Church RM, Eds. Punishment and aversive behavior. New York: Appleton-Century-Crofts 1969; pp. 279-96.

[22] Holland PC. Occasion-setting in Pavlovian feature positive discriminations. In: Commons ML, Herrnstein RJ, Wagner AR, Eds. Quantitative analyses of behavior: discrimination processes. New York: Ballinger 1983.

[23] Holland PC. Transfer of negative occasion setting and conditioned inhibition across conditioned and unconditioned stimuli. J Exp Psychol Anim Behav Process 1989; 15(4): 311-28.

[24] Brooks DC, Bouton ME. A retrieval cue for extinction attenuates spontaneous recovery. J Exp Psychol Anim Behav Process 1993; 19(1): 77-89.

[25] Brooks DC, Bowker JL. Further evidence that conditioned inhibition is not the mechanism of an extinction cue's effect: A reinforced prevents spontaneous recovery. Anim Learn Behav 2001; 29(4): 381-8.

[26] Vansteenwegen D, Vervliet B, Hermans D, Beckers T, Baeyens F, Eelen P. Stronger renewal in human fear conditioning when tested with an acquisition retrieval cue than with an extinction retrieval cue. Behav Res Ther 2006; 44(12): 1717-25.

[27] Collins BN, Brandon TH. Effects of extinction context and retrieval cues on alcohol cue reactivity among nonalcoholic drinkers. J Consult Clin Psychol 2002; 70(2): 390-7.

[28] Weijters B, Geuens M, Schillewaert N. The stability of individual response styles. Psychol Methods 2010; 15(1): 96-110.

[29] Eisenstein EM, Eisenstein D, Bonheim P, Welch EA. Habituation of the galvanic skin response in adult males as a function of age. Physiol Behav 1990; 48(1): 169-73.

[30] Halpern DL, Blake R, Hillenbrand J. Psychoacoustics of a chilling sound. Percept Psychophys 1986; 39(2): 77-80.

Received: May 31, 2012

(C) Dibbets et al.; Licensee Bentham Open.

This is an open access article licensed under the terms of the Creative Commons Attribution Non-Commercial License (http://creativecommons.org/licenses/ by-nc/3.0/) which permits unrestricted, non-commercial use, distribution and reproduction in any medium, provided the work is properly cited. 